\title{
c-FLIP is a target of the E3 ligase deltex1 in gastric cancer
}

\author{
Tzu-Sheng Hsu', Shu-Ting Mo', Ping-Ning Hsu² and Ming-Zong Lai $\mathbb{B}^{1,2}$
}

\begin{abstract}
The ubiquitin E3 ligase DELTEX1 (DTX1) is specifically downregulated in gastric cancer tissues, and expression of DTX1 is linked to better prognoses and survival in gastric cancer. Cellular FLICE inhibitory protein (C-FLIP) is known for its pivotal role in the resistance of cancer cells to death receptor-induced cell death. Here, we show that DTX1 is an E3 ligase for c-FLIP in gastric cancer cells. DTX1 promoted c-FLIP downregulation. Overexpression of DTX1 sensitized gastric cancer cells to TRAIL-induced apoptosis, whereas DTX1-knockdown attenuated apoptosis induction. DTX1 binds C-FLIP and directs it into the endosome-lysosomal pathway for proteasome-independent degradation. Moreover, induction of DTX1 in AGS cells by geldanamycin conferred susceptibility of those cells to TRAIL-induced apoptosis. Our results reveal a tumor-suppressive role for DTX1 and suggest a new approach to increasing TRAIL efficacy by raising DTX1 levels in gastric cancer therapy. DTX1 also enhanced c-FLIP degradation and FasL-induced and TRAIL-induced apoptosis in T cells, suggesting that DTX1 constitutes one of the physiological mechanisms regulating c-FLIP stability.
\end{abstract}

\section{Introduction}

Gastric cancer cells are characterized by their resistance to apoptosis induction by death receptors. Gastric cancer has one of the world's leading cancer mortality rates, with a poor 5 -year survival rate ${ }^{1-3}$. Advanced stages of gastric cancer show local invasion, peritoneal dissemination, and hepatic or para-aortic lymph node metastasis. Surgery remains the curative therapy, but is limited to nonmetastatic gastric cancer. The efficacy of chemotherapy for gastric cancer is poor due to multidrug resistance (MDR). Therefore, identification of novel biomarkers and development of new therapeutics for gastric cancer are one of the demanding priorities.

The Death receptor (DR) agonist TRAIL has been explored for its efficacy to induce apoptosis in different types of cancers ${ }^{4-6}$, including gastric cancer ${ }^{7,8}$. Like other death receptors, engagement of TRAIL receptors (DR4 and DR5) by TRAIL results in the formation of death-

\footnotetext{
Correspondence: M-Z. Lai (mblai@gate.sinica.edu.tw)

${ }^{1}$ Institute of Molecular Biology, Academia Sinica, Taipei, Taiwan

${ }^{2}$ Institute of Immunology, College of Medicine, National Taiwan University,

Taipei, Taiwan

Edited by T. Kaufmann
}

inducing signaling complexes (DISC) containing FADD and procaspase- $8^{9-12}$. Procaspase- 8 undergoes autoproteolytic cleavage to generate active caspase- 8 at DISC, leading to activation of downstream caspases and irreversible cell damage. Cellular FLICE-inhibitory protein (cFLIP) is a master anti-apoptotic factor that suppresses death receptor-induced apoptosis by interfering with the processing of procaspase-8 at DISC ${ }^{9-15}$. C-FLIP also inhibits necrosis and autophagy ${ }^{16-18}$. c-FLIP is partly accountable for the failure of TRAIL receptor agonists in clinical attempts to treat cancers ${ }^{4,19}$, so it is a target for cancer therapy ${ }^{19-21}$. Expression of c-FLIP is induced by activation signaling, including NF- $\mathrm{KB}^{22}, \mathrm{Akt}$, and ERK $^{13,19,22-24}$. Levels of c-FLIP protein are subjected to regulation by two ubiquitin E3 ligases, ITCH and CBL, through the promotion of polyubiquitination and subsequent proteosomal degradation of c-FLIP ${ }^{25,26}$.

TRAIL receptors and the downstream effector caspase8 are intact in gastric cancer cells ${ }^{27,28}$. However, gastric cancers are generally resistant to TRAIL-induced cell death, and induction of TRAIL-mediated cytotoxicity always requires co-stimulation with a sensitizing reagent. 
c-FLIP is upregulated in gastric cancer and is associated with metastasis and tumor progression ${ }^{29,30}$. As in other types of cancer, c-FLIP contributes to the resistance to TRAIL-induced apoptosis in gastric cancer ${ }^{31-34}$. We have previously shown that Helicobacter pylori enhances the susceptibility to TRAIL-induced apoptosis in gastric cancer cells by downregulation of c-FLIP ${ }^{34}$.

Deltex (DTX) is a target of Notch, and is composed of Notch-binding WWWE domains at the N-terminus, followed by a proline-rich motif, and a C-terminal RING finger domain ${ }^{35,36}$. DTX1 confers ligand-independent activation of Notch by directing the ubiquitination and endosomal entry of Notch ${ }^{37,38}$. Similar to the E3 ligases Itch and $\mathrm{Cbl}^{3} \mathrm{~b}^{39}$, DTX1 is a target of NFAT and is involved in $\mathrm{T}$ cell tolerance ${ }^{40,41}$. We recently found that DTX1 promotes the degradation of PKC $\theta$ and PLC- $\gamma$ in a way similar to ITCH and Cbl-b ${ }^{42}$.

In the present study, we show that DTX1 is specifically downregulated in gastric cancer and is critical for the resistance of gastric cancer cells to TRAIL-induced cell death. DTX1 binds to c-FLIP and promotes degradation of c-FLIP through the endosome-lysosomal pathway. Reintroduction of DTX1 into gastric cancer cells increased TRAIL-induced apoptosis and also reduced c-FLIP. In addition, a treatment that increased DTX1 expression also sensitized gastric cancer to TRAIL treatment. Our results suggest that induction of DTX1 could be a new approach to enhancing the benefits of TRAIL-mediated cancer therapy. We also found that DTX1 enhanced c-FLIP degradation and Fas-induced and TRAIL-induced apoptosis in T cells, indicating that DTX1 constitutes one of the physiological mechanisms regulating c-FLIP stability.

\section{Results}

DTX1 expression is negatively correlated with gastric cancer progression

We found that expression of the ubiquitin E3 ligase DELTEX1 (DTX1) is reduced in gastric adenocarcinoma tissues from patients (Fig. 1a). In contrast, expression of $I T C H$ and $C B L$, the ubiquitin E3 ligases that have been shown to promote polyubiquitination and proteosomal degradation of c-FLIP ${ }^{25,26}$, are normal or increased in the same gastric cancer tissues (Fig. 1b, c) ${ }^{43-45}$. In another analysis of human gastric cancer cell lines ${ }^{45}$, expression of $D T X 1$ is reduced in most of the gastric cancer cell lines examined (Fig. 1d). Expression of $C B L$ is variable in different gastric cancer cell lines, whereas the expression of ITCH is increased in the same group of gastric cancer cell lines (Fig. 1e, f), suggesting that the ITCH-mediated and CBL-mediated c-FLIP degradation processes are not operational in gastric cancer. Gene expression-based prognosis risk score analyses in gastric cancer have also shown that gastric cancer tissues from relapse-free survival (RFS) patients ${ }^{46}$ expressed higher levels of DTX1
mRNA (Fig. 1g, h). This is in contrast to no correlation being found between the expression of $\mathrm{CBL}$ or $I T C H$ and the RFS of gastric cancer patients (Fig. 1i, j). Therefore, $D T X 1$ is downregulated in gastric cancer tissues and DTX1 expression is negatively correlated with gastric cancer progression.

\section{DTX1 promotes c-FLIP downregulation and TRAIL-induced apoptosis in gastric cancer cells}

DTX1 is a ubiquitin E3 ligase that is functionally analogous to ITCH and CBL in T cell anergy ${ }^{40,42}$. Since ITCH and CBL stimulate c-FLIP downregulation, we examined the possible effect of DTX1 on levels of c-FLIP ${ }_{\mathrm{L}}$. We found that overexpression of DTX1 in human gastric adenocarcinoma AGS cells decreased the levels of c-FLIP ${ }_{\mathrm{L}}$

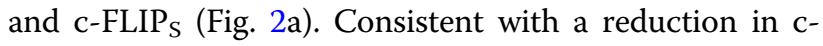
FLIP, DTX1-expressing AGS cells were more sensitive to apoptosis induced by TNF-related apoptosis-inducing ligand (TRAIL) (Fig. 2a). In another gastric cancer cell line, SNU-16, DTX1 expression also reduced the protein levels of FLIP $_{L}$ and c-FLIP , with a concomitant increase in TRAIL-induced cell death (Fig. 2b). This effect was accompanied by enhanced activation of pro-caspase- 8 into p43 and p18 in DTX1-expressing AGS cells (Fig. 2c). Enhanced formation of active caspase-3 was also found in TRAIL-stimulated DTX1-expressing AGS cells (Fig. 2c).

DTX1 expression did not affect mRNA expression of cFLIP (Supplementary Fig. 1a), nor did it affect the levels of Mcl-1, Bcl-2, or caspase-8 (Supplementary Fig. 1b). Even though total concentrations of the TRAIL receptors DR4 and DR5 were increased in DTX1-expressing AGS cells, cell surface levels of DR4 and DR5 remained unchanged (Supplementary Fig. 1b). In contrast, DTX1 increased cFLIP $_{\mathrm{L}}$ protein instability (Fig. $2 \mathrm{~d}$ ), with co-expression of DTX1 decreasing the half-life of $\mathrm{c}-\mathrm{FLIP}_{\mathrm{L}}$ protein by $50 \%$ (Fig. 2e).

SNU-16 cells are different from AGS cells due to their detectable expression levels of endogenous DTX1, allowing us to address the role of DTX1 by its knockdown. Levels of FLIP $_{\mathrm{L}}$ and $\mathrm{C}-F L I P_{\mathrm{S}}$ proteins were increased in DTX1-knockdown SNU-16 cells, consistent with a reduction in TRAIL-induced apoptosis (Supplementary Fig. 2). Together, these results suggest that DTX1 enhances DR-induced apoptosis through downregulation of FLIP $_{\mathrm{L}}$.

\section{DTX1 interacts with c-FLIP}

We next examined how DTX1 regulates the stability of c-FLIP protein. We found that DTX1 promoted downregulation of c-FLIP ${ }_{L}$-FLAG and c-FLIP ${ }_{S}$-FLAG in 293T cells (Fig. 3a). Immunoprecipitation of DTX1myc brought down c-FLIP L-FLAG and $\mathrm{p} 43$ c-FLIP $_{\mathrm{L}}$-FLAG, but not c-FLIP -FLAG (Fig. 3b). The c-FLIP caspase-like domain (CLD) was identified as the DTX1-interacting 


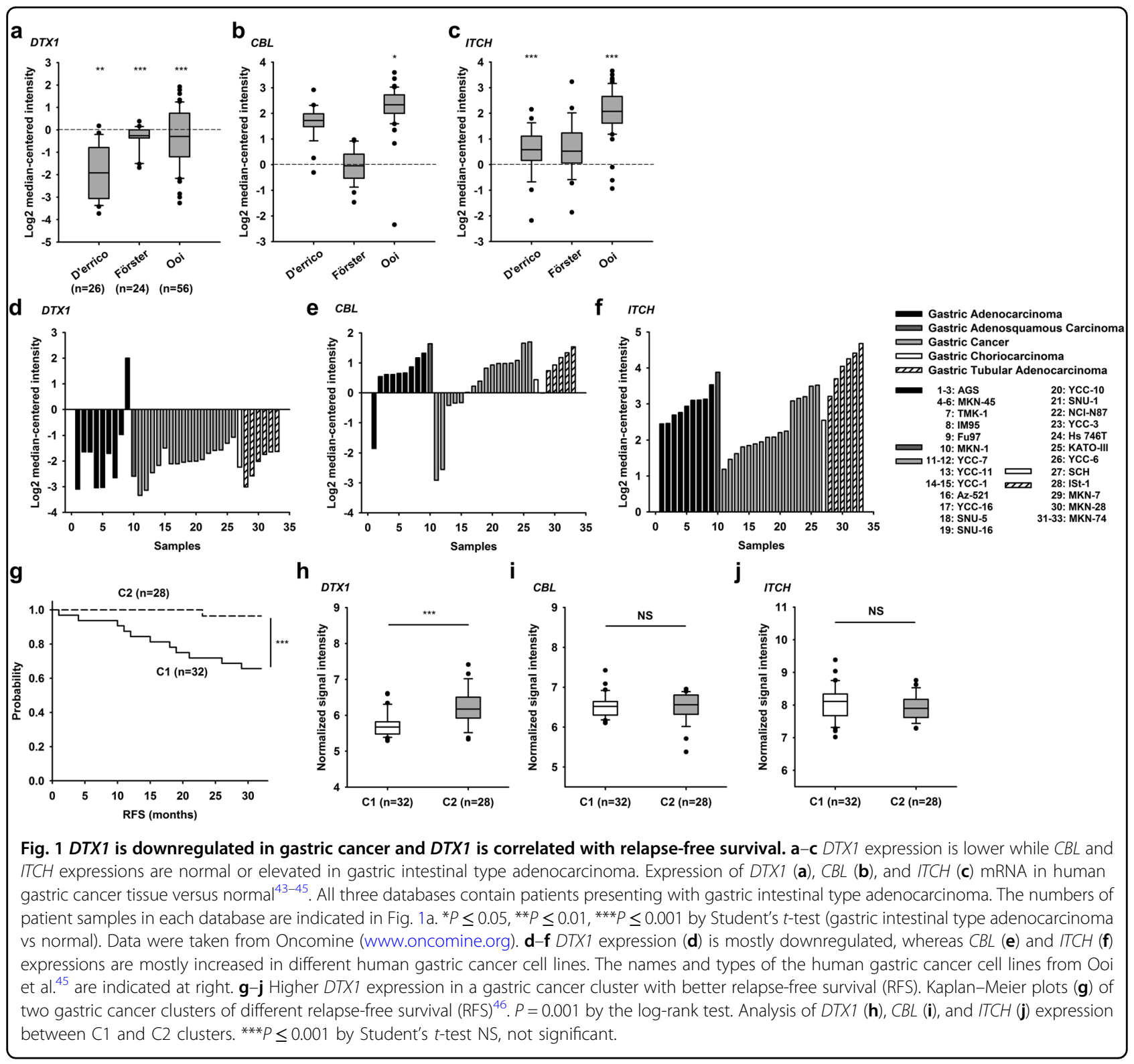

region, evidenced by the ability of DTX1 to bind the CLD (Fig. 3b). We further demonstrated that p20 of the CLD was pulled down by DTX1 (Fig. 3c), suggesting that p20 of the CLD is the region on c-FLIP that binds DTX1. Therefore, DTX1 binds c-FLIP $\mathrm{L}_{\mathrm{L}}$ but does not interact with c-FLIP . We then investigated how DTX1 enhances the down-regulation of c-FLIP ${ }_{S}$ in $293 \mathrm{~T}$ cells (Fig. 3a). Only c-FLIP ${ }_{L}$ was expressed in 293T cells (Supplementary Fig. 3a). When transfected in higher amounts into 293T cells, c-FLIP was resistant to DTX1-mediated downregulation (Supplementary Fig. 3b), in agreement with the inability of c-FLIP to $_{\mathrm{S}}$ bind DTX1. However, c-FLIP ${ }_{S}$-FLAG was downregulated by

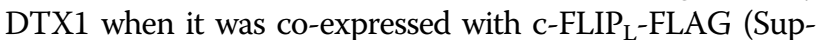
plementary Fig. 3c). Immunoprecipitation of DTX1-myc also pulled down c-FLIP ${ }_{S}$-FLAG when c-FLIP ${ }_{L}$-FLAG was co-expressed (Supplementary Fig. 3d). In addition, on introduction of c-FLIP ${ }_{S}$-FLAG alone, it was found to associate with endogenous c-FLIP $\mathrm{L}_{\mathrm{L}}$ in 293T cells (Supplementary Fig. 3e). These results suggest that intracellular hetero-dimerization of c-FLIP $\mathrm{S}_{\mathrm{S}}$ with $\mathrm{c}-\mathrm{FLIP}_{\mathrm{L}}$ leads to susceptibility of both c-FLIP and c-FLIP ${ }_{\mathrm{L}}$ to DTX1-mediated downregulation in vivo (Fig. 2a, b).

\section{DTX1 promotes C-FLIP $\mathrm{L}_{\mathrm{L}}$ degradation by the endosome- lysosome-dependent pathway}

DTX1-mediated c-FLIP $\mathrm{L}_{\mathrm{L}}$ degradation was not prevented by addition of the proteosome inhibitor MG132 (Fig. 4a), suggesting that proteasomes are not involved in this process. 


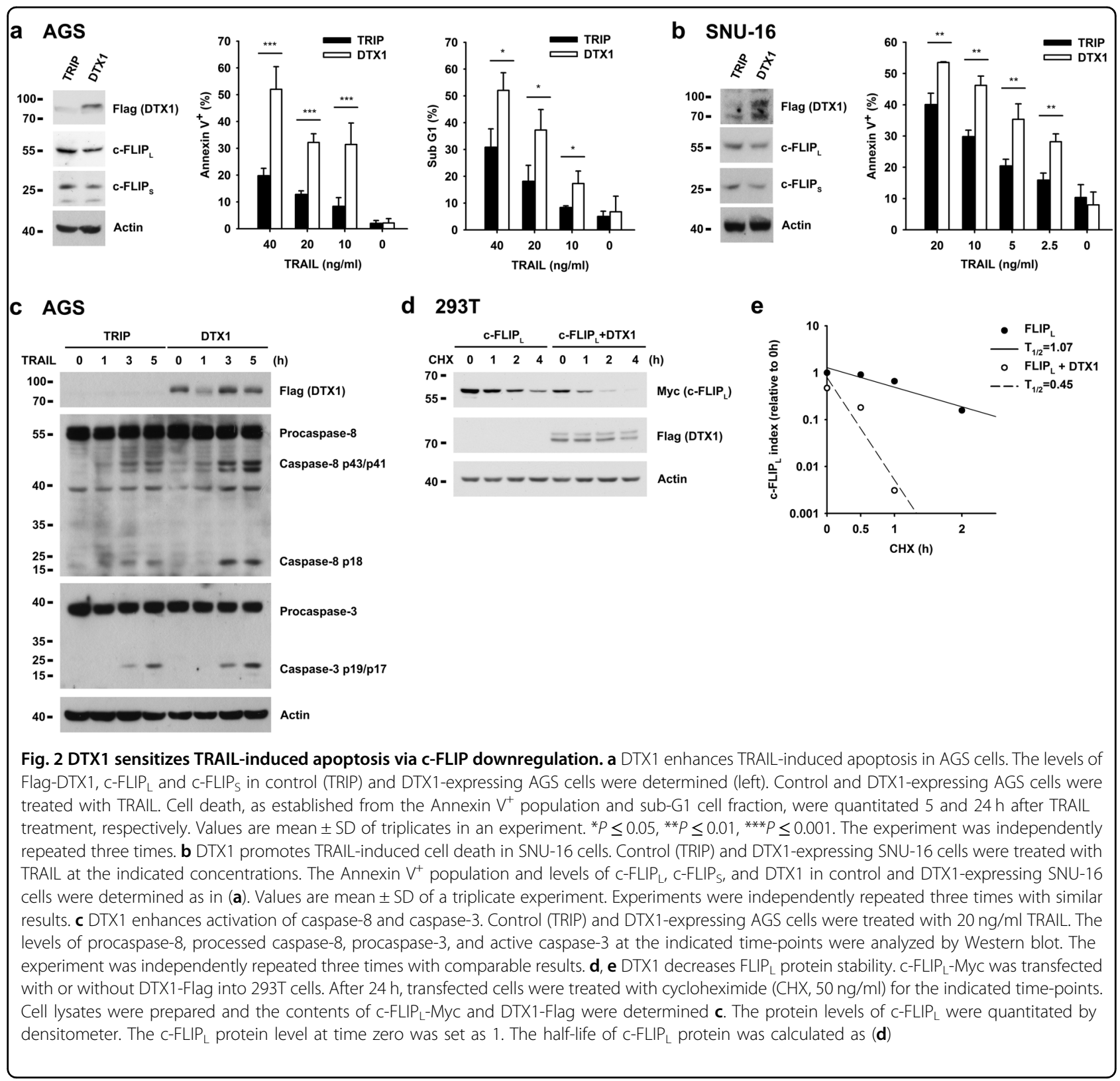

Addition of $\mathrm{NH}_{4} \mathrm{Cl}$ or leupeptin partially protected c-FLIP $\mathrm{L}$ from DTX1-induced degradation (Fig. 4a), indicating the involvement of endosome-lysosomal pathways. To further determine the requirement of E3 ligase activity of DTX1 in cFLIP degradation, we used DTX1 with mutation at E3 ligase active sites (H453N and H456N, H2N2) and found that

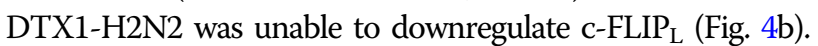

c-FLIP $\mathrm{L}_{\mathrm{L}}$ alone was evenly distributed in the nucleus and cytoplasm, without apparent co-localization with the early endosome marker Rab5 (Fig. 4c). Coexpression of DTX1 and $\mathrm{c}-\mathrm{FLIP}_{\mathrm{L}}$ resulted in a significant increase in $\mathrm{c}-\mathrm{FLIP}_{\mathrm{L}}$ and Rab5 co-localization (Fig. 4c), suggesting that entry of $\mathrm{c}-\mathrm{FLIP}_{\mathrm{L}}$ into endosomal compartments is promoted by the c-FLIP $\mathrm{L}^{-}$ DTX1 association. Similarly, c-FLIP ${ }_{L}$ was not located in lysosomes when expressed alone, yet the presence of DTX1 enhanced co-localization of c-FLIP ${ }_{L}$ with the lysosomal marker LAMP (Fig. 4c). Together, these results suggest that DTX1 directs $\mathrm{c}-\mathrm{FLIP}_{\mathrm{L}}$ into endosomal and lysosomal compartments for c-FLIP degradation.

\section{Geldanamycin decreases the protein level of c-FLIP and enhances TRAIL sensitivity}

We next examined whether the DTX1-mediated downregulation of c-FLIP contributes to sensitization of 
cancer cells to TRAIL-induced cell death. Geldanamycin (GA) induces c-FLIP ${ }_{\mathrm{L}}$ degradation and cellular apoptosis in human lung cancer cells ${ }^{47}$. Treatment of AGS and SNU-16 cells with GA for $24 \mathrm{~h}$ also resulted in c-FLIP $\mathrm{L}$ downregulation (Fig. 5a, b). Addition of GA also enhanced the ability of TRAIL to induce cell death in AGS cells (Fig. 5c). We found that GA treatment led to increased DTX1 protein levels in AGS and SNU-16 cells (Fig. 5a, b). Notably, GA treatment did not affect the expression of DTX1 or c-FLIP mRNA (Supplementary Fig. 4).

We then examined whether DTX1 was involved in GA-induced TRAIL sensitivity and c-FLIP degradation. DTX1-knockdown did not affect apoptosis induced by TRAIL alone in AGS cells, likely due to low DTX1 expression (Fig. 6a), but DTX1-deficiency reduced apoptosis triggered by TRAIL plus GA (Fig. 6a). The celldeath triggered by the combination of TRAIL and GA was similarly attenuated by DTX1-knockodwn in SNU16 cells (Fig. 6b). GAinduced c-FLIP degradation was prevented by DTX1-knockdown in AGS cells (Fig. 6c). These results suggest that the GA-induced c-FLIP degradation was partly attributable to the induction of DTX1, leading to enhanced sensitivity of gastric cancer cells to TRAIL treatment. Together, our results suggest that increased DTX1 enhances TRAIL-triggered apoptosis in gastric cancer cells partly by downregulation of c-FLIP.

\section{DTX1 enhances FasL-induced and TRAIL-induced apoptosis in $T$ cells}

The involvement of DTX1 in c-FLIP degradation is not limited to gastric cancer cells. We used $D t x 1^{-/-}$ $\mathrm{T}$ cells to examine the role of DTX1 in c-FLIP downregulation. c-FLIP was barely detectable in naïve WT $\mathrm{T}$ cells or $D t x 1^{-1-} \mathrm{T}$ cells. TCR stimulation induced the expression of c-FLIP, and DTX1-deficiency increased CD3-triggered c-FLIP expression (Supplementary Fig. 5). Activated Dtx $1^{-1-} \mathrm{T}$ cells were more resistant to FasL-induced apoptosis. We further explored whether overexpression of DTX1 increased the sensitivity of $\mathrm{T}$ lymphoma to death receptorinduced cell death by targeting c-FLIP ${ }_{\mathrm{L}}$ for degradation. DTX1 expression reduced the levels of c-FLIP in Jurkat cells (Fig. 7a). Increased DTX1 expression was indeed accompanied by enhanced FasL-induced apoptosis and TRAIL-triggered cell death in Jurkat cells (Fig. 7b, c). In addition, increased DTX1 expression promoted TNF- $\alpha$-induced apoptosis in Jurkat cells in the presence of cycloheximide (Fig. $7 \mathrm{~d}$ ). Thus, DTX1 promotes DR-induced cell death in
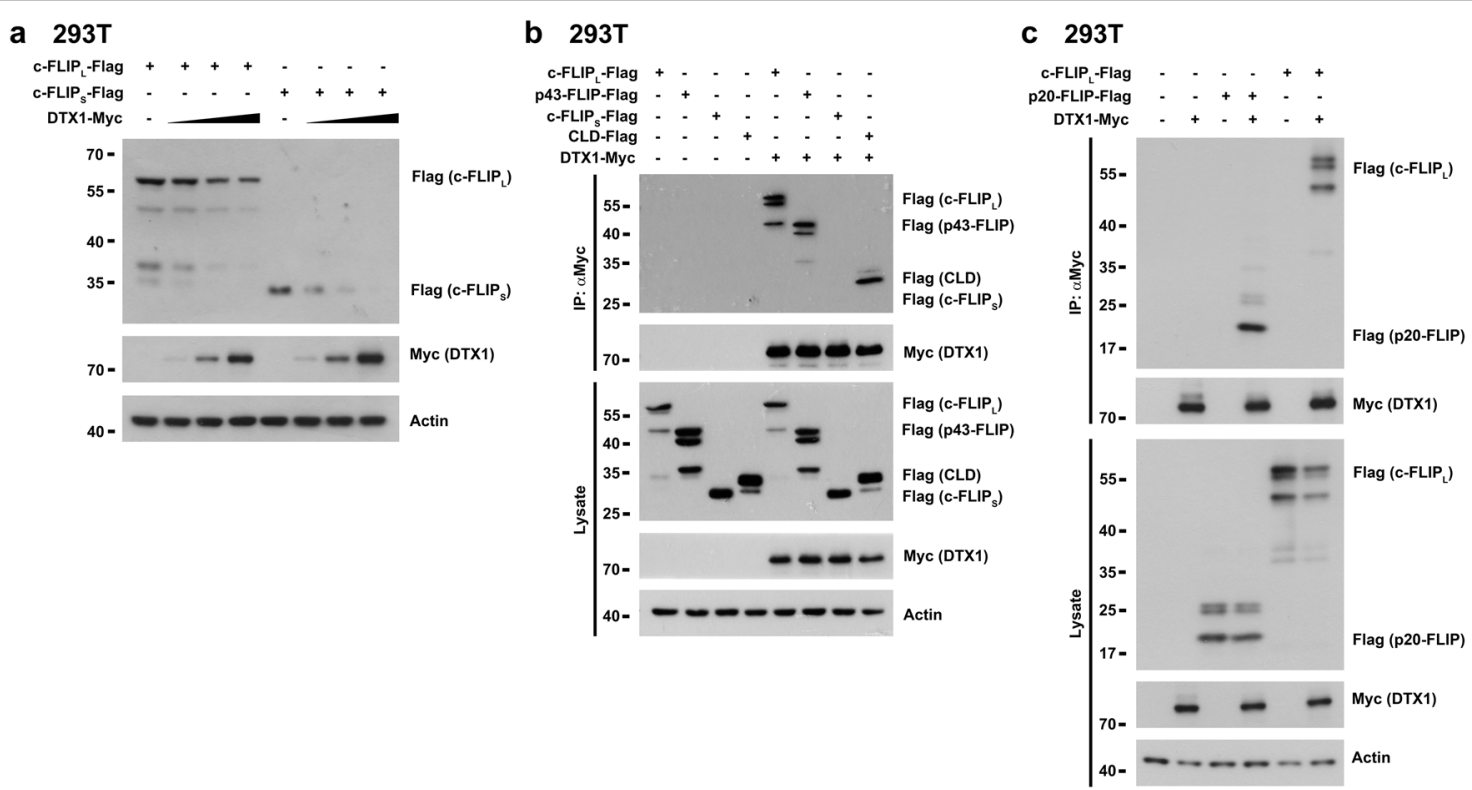

Fig. 3 DTX1 binds c-FLIP $\mathrm{L}_{\mathrm{L}}$ and promotes c-FLIP downregulation. a DTX1 promotes C-FLIP degradation. 293T cells were transfected with c-FLIP $\mathrm{L}^{-}$ Flag (200 ng) or c-FLIPS-Flag (2 ng) with increasing amounts of DTX1-Myc. The levels of DTX1, c-FLIP and c-FLIPS were determined $24 \mathrm{~h}$ after transfection. $\mathbf{b}$ The caspase-like domain of c-FLIP binds DTX1. DTX1-Myc, c-FLIP, c-FLIP-p43, c-FLIPS and the caspase-like domain (CLD) were transfected into 293T cells as indicated. Cell lysates were prepared $24 \mathrm{~h}$ later and were immunoprecipitated by anti-Myc. The presence of DTX1 and different forms of c-FLIP in the precipitates and lysates were determined by anti-Myc and anti-Flag. $\mathbf{c}$ The p20 region in the CLD of c-FLIP binds DTX1. DTX1-Myc, c-FLIPL, and c-FLIP-p20 were transfected into 293T cells as indicated. The levels of DTX1 and different forms of c-FLIP in lysates and antiMyc immunoprecipitates were determined by anti-Myc and anti-Flag. Experiments (a-c) were independently repeated three times with similar results 


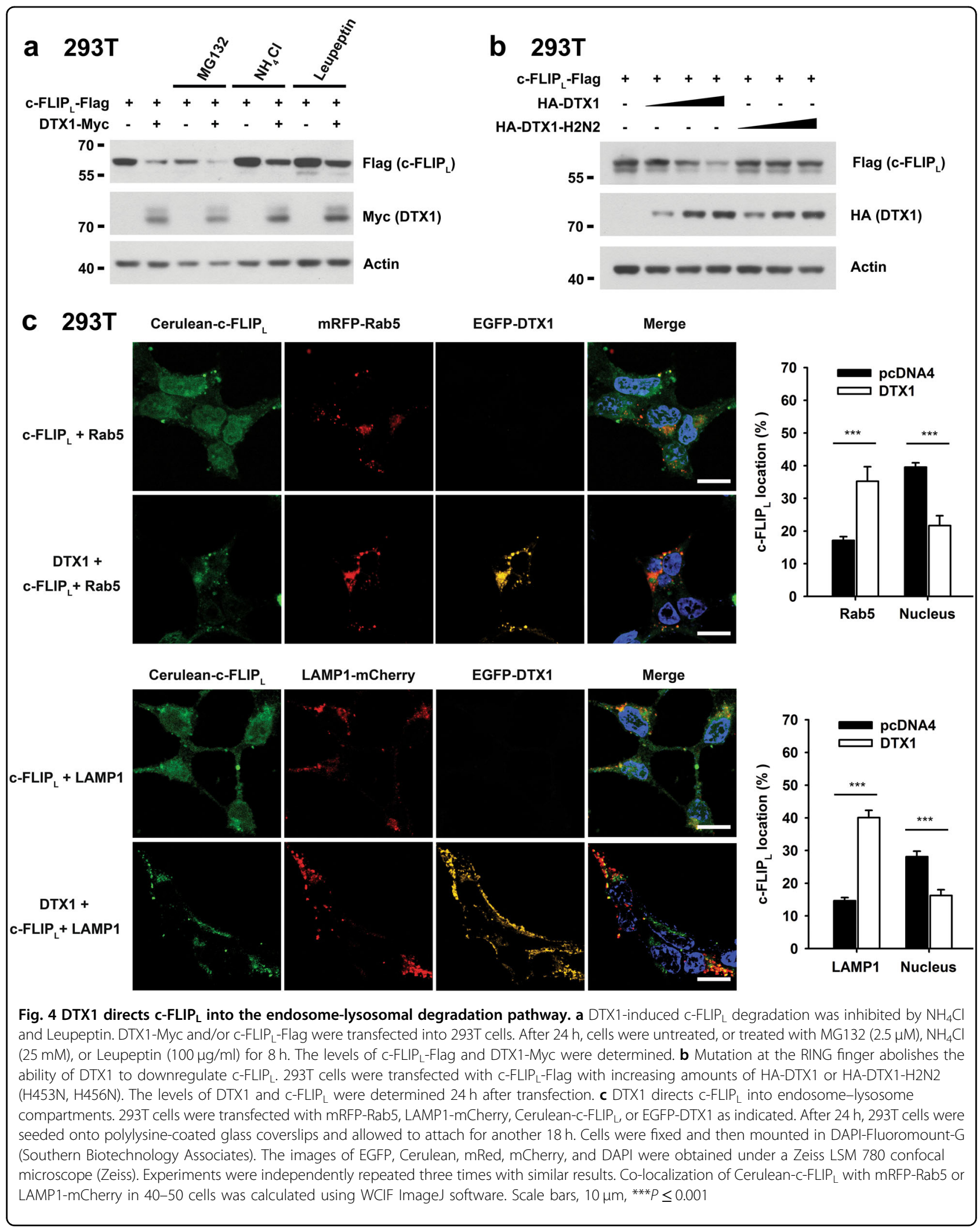


normal $\mathrm{T}$ cells and in T lymphoma. DTX1-promoted c-FLIP instability represents one of the physiological mechanisms that regulate c-FLIP protein.

\section{Discussion}

Here, we report a new finding that DTX1 is an E3 ligase for c-FLIP in gastric cancer cells. The presence of DTX1 profoundly reduced the protein stability of c-FLIP (Fig. 2d, e). We have previously demonstrated that DTX1, similar to Cbl-b and Itch, promotes PKC $\theta$ and PLC- $\gamma$ degradation in anergic Th1 cells ${ }^{42}$. In addition, as for Cbl-b and $\mathrm{Itch}^{39}$, DTX1 induced PKC $\theta$ degradation by directing it to the endocytic pathway for lysosomal degradation. We illustrate that DTX1 promotes c-FLIP $\mathrm{L}_{\mathrm{L}}$ protein downregulation by similarly directing c-FLIP $\mathrm{L}_{\mathrm{P}}$ into the endosome-lysosomal pathway for degradation (Fig. 4). Consequently, DTX1 expression enhanced the susceptibility of gastric cancer cells to TRAIL-induced apoptosis (Fig. 2a, b), whereas DTX1 downregulation increased the resistance to TRAILtriggered cell death (Supplementary Fig. 2).

We have demonstrated that DTX1 interacts with cFLIP $_{\mathrm{L}}$ but not with c-FLIP , and reveal that the $\mathrm{p} 20$ region of the caspase-like domain in c-FLIP $\mathrm{L}_{\mathrm{L}}$ is the DTXassociation domain (Fig. 3b, c). Binding of DTX1 to cFLIP $_{\mathrm{L}}$ leads to c-FLIP $\mathrm{L}$ degradation. Notably, expression of DTX1 led to a reduction of both c-FLIP ${ }_{L}$ and c-FLIP levels in AGS, SNU-16, and Jurkat cells (Figs 2a, b and 7a). A likely cause for the downregulation of c-FLIP ${ }_{S}$ in these cancer cells is that c-FLIP $\mathrm{L}_{\mathrm{L}}$ is hetero-dimerized with cFLIP $_{\mathrm{S}}$ in vivo and that the binding of DTX1 to c-FLIP $\mathrm{L}_{\mathrm{L}}$ induces the degradation of $\mathrm{c}-$ FLIP $_{\mathrm{L}}$-c-FLIP $\mathrm{P}_{\mathrm{S}}$ dimers. cFLIP $_{\mathrm{S}}$ was associated with the endogenous $\mathrm{c}^{-\mathrm{FLIP}_{\mathrm{L}}}$ in 293T cells (Supplementary Fig. 3e). This is further supported by the fact that DTX1 is co-expressed with cFLIP $_{\mathrm{L}}$ and c-FLIP $\mathrm{S}$ dimers in 293T cells, resulting in downregulation of both c-FLIP ${ }_{L}$ and c-FLIP (Fig. 3a, Supplementary Fig. 3c), and by the observation that cFLIP $_{S}$ is associated with DTX1 under co-expression of cFLIP $_{\mathrm{L}}$ (Supplementary Fig. 3d).

DTX1 was downregulated in most of our gastric cancer samples (Fig. 1). Increased expression of c-FLIP in DTX1deficient cells suggests that DTX1 downregulation confers resistance to TRAIL-induced apoptosis in gastric cancer cells. This finding, together with the negative correlation between cancer prognoses and DTX1 expression levels (Fig. 1g, h), suggest that manipulation of DTX1 could be beneficial in therapies against gastric cancer. We propose that reagents that can stimulate the expression of DTX1 may enhance the susceptibility of gastric cancer to TRAIL treatment.

Interaction with HSP90 contributes to the protein stability of c-FLIP. HSP90 also recruits c-FLIP to DISC to inhibit TRAIL-induced cell death ${ }^{48}$. HSP-90 inhibitors are known to exhibit inhibitory effects on multiple signaling pathways in cancer cells ${ }^{49}$. These inhibitors act synergistically with TRAIL to induce cell death in various cancers $^{50-52}$. As reported for gastric cancer ${ }^{53}$, we found that addition of GA augmented TRAIL-induced apoptosis (Fig. 5). We have further demonstrated that DTX1 partly mediates the enhancing effect of GA on TRAIL-induced apoptosis (Figs 5 and 6). Knockdown of DTX1 partly reversed the enhancing effect of GA in AGS and SNU-16 cells (Fig. 6). Even though the exact molecular mechanism requires further investigation, it should be noted that other deltex family members (DTX2, DTX3, DTX4) are also associated with HSP90.
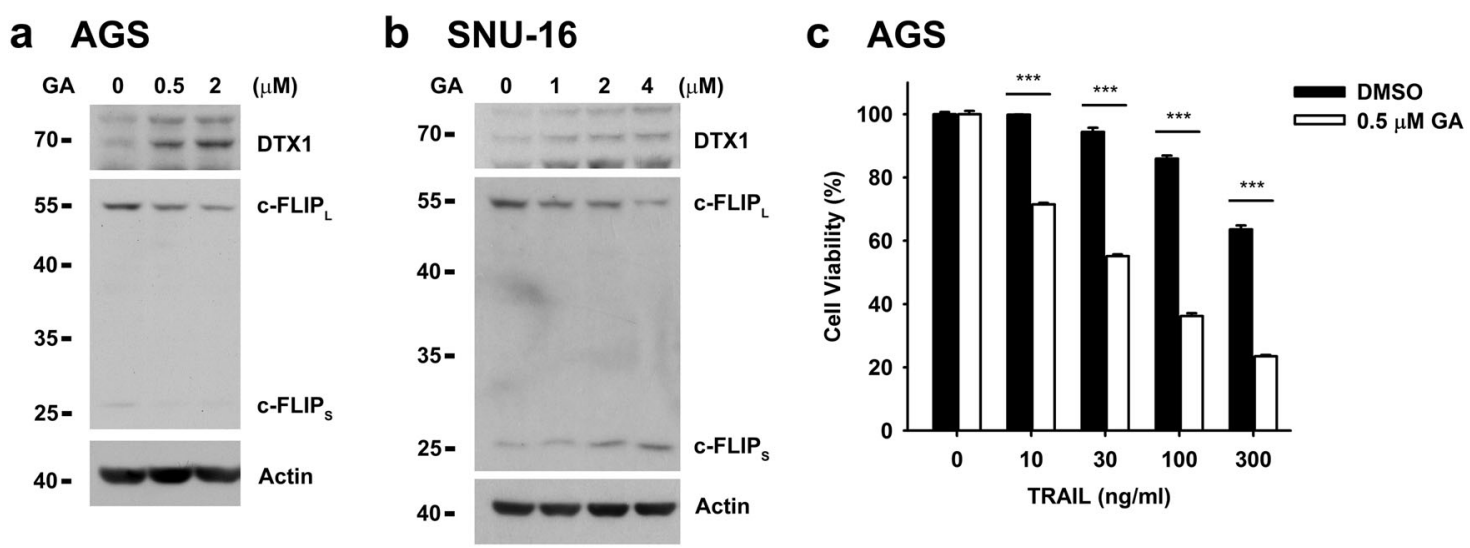

Fig. 5 Geldanamycin decreases the protein level of c-FLIP and enhances TRAIL sensitivity. $\mathbf{a}, \mathbf{b}$ Geldanamycin (GA) promotes C-FLIP degradation. AGS cells (a) or SNU-16 (b) cells were treated with GA at the indicated concentrations for $24 \mathrm{~h}$. The levels of DTX1, C-FLIPL, and C-FLIPS were determined by immunoblotting. c GA sensitizes AGS cells to TRAIL-induced apoptosis. AGS cells were treated with $0.5 \mu M$ GA overnight and then incubated with TRAIL for $24 \mathrm{~h}$. Cell viability was determined by MTT assay. Values are means \pm standard deviations of three independent experiments. ${ }^{* * *} P \leq 0.001$ 

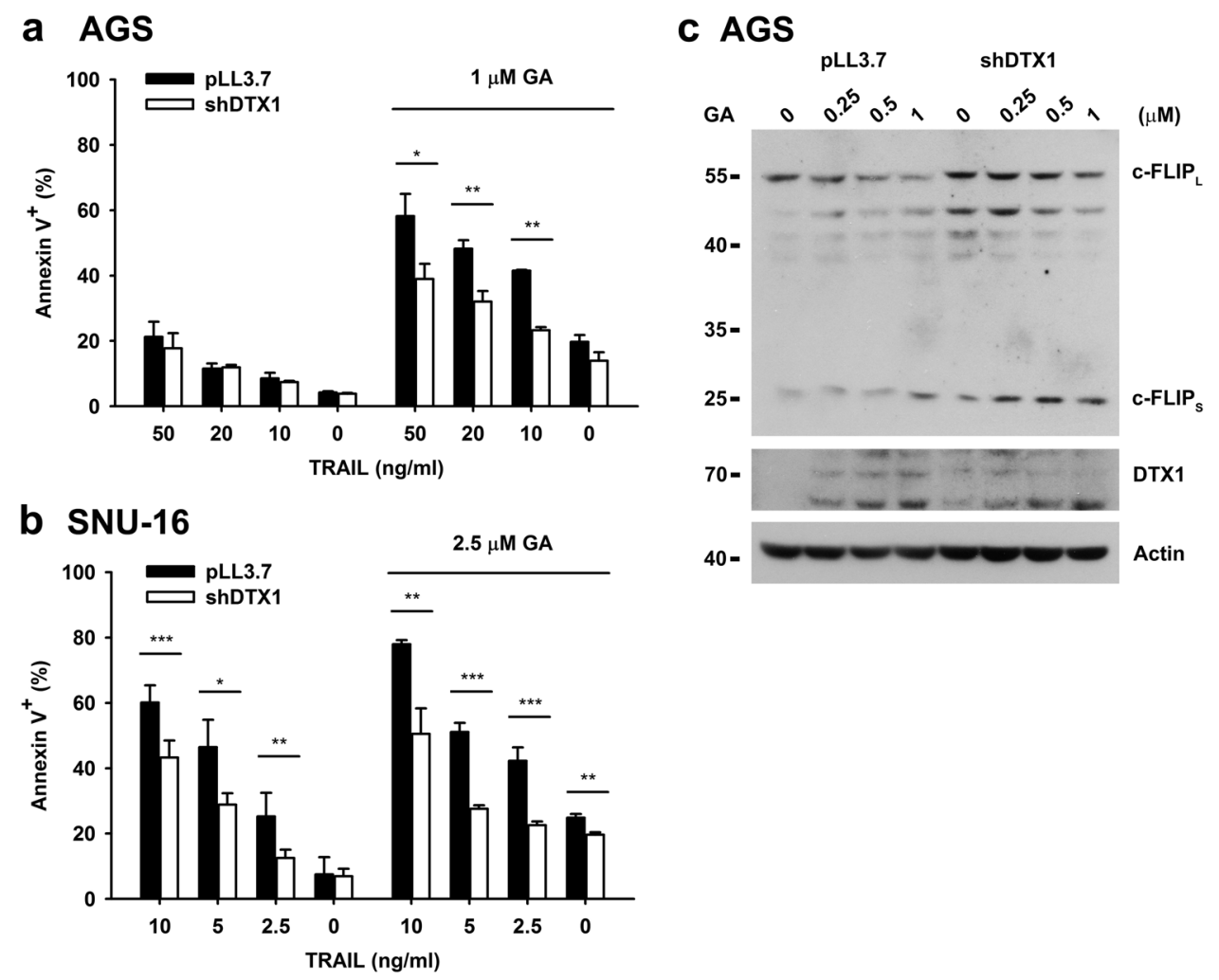

Fig. 6 Geldanamycin enhances TRAIL-induced apoptosis through DTX1-mediated c-FLIPL degradation. a, b DTX1 knockdown reduces GAenhanced TRAIL sensitivity. Control (pLL3.7) and DTX1-knockdown (shDTX1) AGS cells (a) or SNU-16 cells (b) were incubated with GA overnight followed by TRAIL treatment for $5 \mathrm{~h}$. Apoptosis was determined by Annexin V/PI staining. Values are means \pm standard deviations of an experiment with triplicate (a) or three independent experiments $(\mathbf{b})$. ${ }^{*} P \leq 0.05,{ }^{* *} P \leq 0.01,{ }^{*} * P \leq 0.001$. Experiment $(\mathbf{a})$ was repeated three times with similar results. c DTX1-knockdown attenuates GA-induced c-FLIP $P_{L}$ degradation. Control (pLL3.7) and DTX1-knockdown (shDTX1) AGS cells were treated with GA overnight. The levels of DTX1, C-FLIPL, and c-FLIPS were determined. Experiments (c) was independently repeated three times with similar results

Our results suggest variability in the recruitment of a specific E3 ligase and in the mechanistic process of c-FLIP degradation in different cell types. Itch mediates c-FLIP $\mathrm{L}_{\mathrm{L}}$ ubiquitination and degradation in conjunction with JNK signaling in hepatocytes and fibroblasts ${ }^{25}$. CBL has been

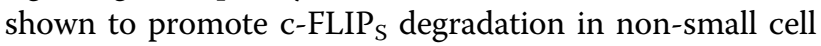
lung carcinoma cells via mTORC2-dependent signaling ${ }^{26}$.

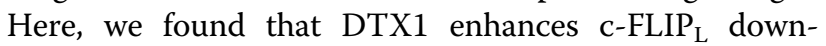
regulation in gastric cancer cells and lymphomas via an endosome-lysosomal pathway. Given the vast heterogeneity between different tumors, selection of E3 ligases for c-FLIP degradation is likely determined by the availability of the given E3 ligase and the signaling within tumor microenvironments. We also illustrate that c-FLIP protein levels are regulated by DTX1 in normal T cells (Supplementary Fig. 5), suggesting that DTX1 represents one of the physiological mechanisms controlling c-FLIP stability. It should be noted that the effect of DTX1deficiency was not overwhelming (Supplementary Figs. 2 and 5), suggesting that DTX1 contributes to but is unlikely to be the only regulator of c-FLIP protein instability.
In summary, we identify DTX1, but not CBL or ITCH, as the E3 ligase regulating C-FLIP protein stability in gastric cancer cells. In addition, we show that DTX1 joins ITCH and CBL as another E3 ligase controlling c-FLIP under physiological conditions. Different c-FLIP degradation pathways are likely to have cancer type-dependent mechanisms, yet to be determined. We have also demonstrated that gastric cancer cells, which are relatively resistant to TRAIL treatment, become susceptible to TRAIL-induced cell death in the presence of DTX1. Our results suggest that a combination of TRAIL with compounds that increase DTX1 expression could be a new approach for gastric cancer therapy.

\section{Materials and methods \\ Reagents}

Recombinant human TRAIL and anti-His were obtained from R\&D Systems (Minneapolis, MN). MG132, propidium iodide (PI), 3-(4,5-dimethyl-thiazol-2-yl)-2,5diphenyl tetrazolium bromide (MTT), and anti-Flag-HRP were purchased from Sigma (St. Louis, MO). Rabbit anti- 

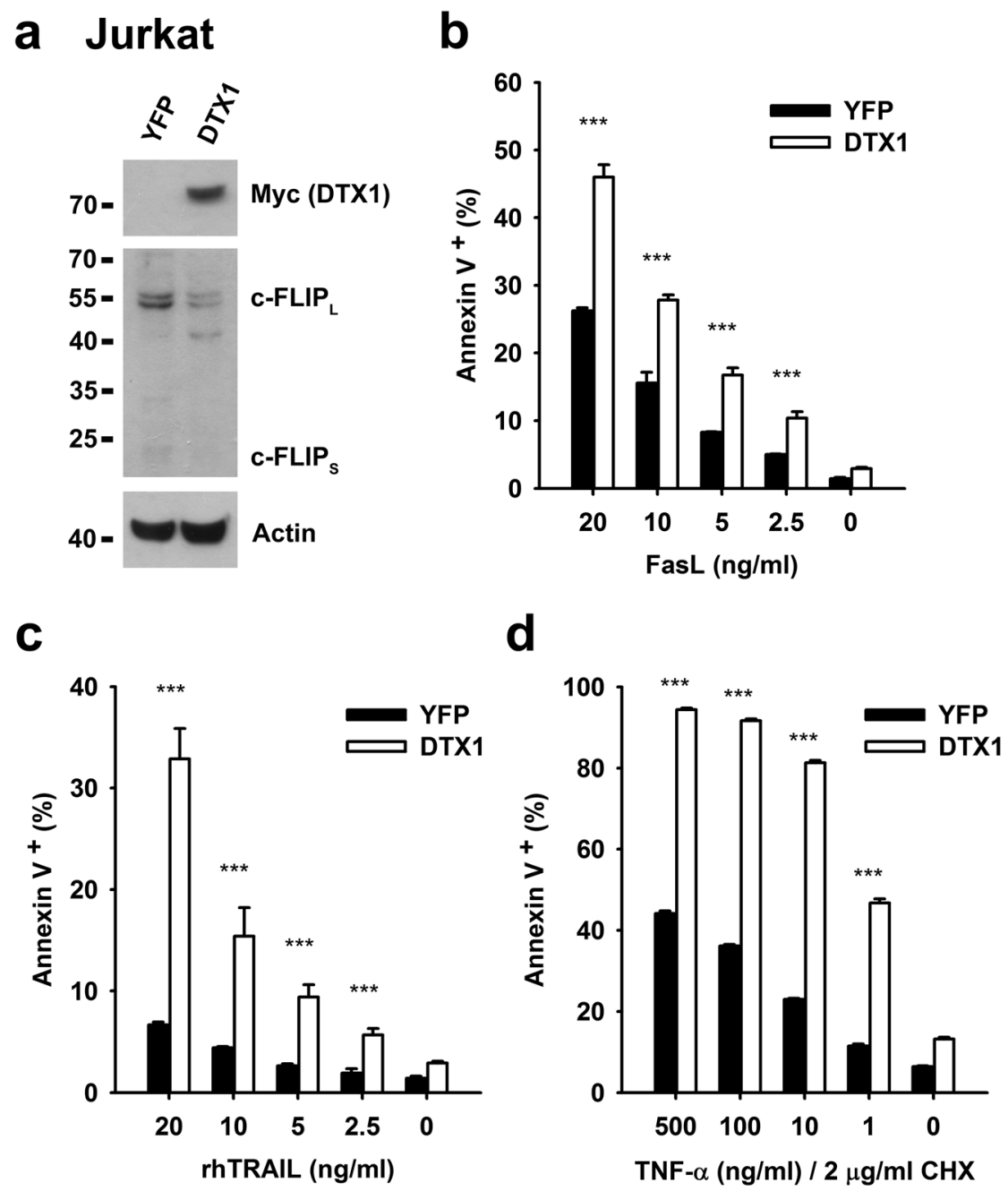

Fig. 7 DTX1-overexpression enhances FasL-induced, TRAIL-induced and TNF-a-induced apoptosis in Jurkat cells. a Reduced c-FLIP levels in DTX1-overexpressing Jurkat cells. The levels of C-FLIP,$c-F L I P_{S}$, and DTX1(Myc) in control (YFP) and DTX1-expressing Jurkat cells were determined. b-d Control (YFP) and DTX1-expressing Jurkat cells were treated with FasL $\mathbf{b}$, TRAIL $\mathbf{c}$, or TNF- $\mathbf{a} \mathbf{d}$ at the indicated concentrations. The extent of apoptosis was analyzed $6 \mathrm{~h}$ later by flow cytometry with Annexin $V$ staining. Data are mean \pm SD of a triplicate experiment. Experiments were independently repeated three times with similar results. ${ }^{* *} P \leq 0.001$. The experiment was independently repeated twice with comparable results

DTX1 polyclonal antiserum against GST-Deltex1 was generated as described ${ }^{9}$. The following antibodies were obtained from Santa Cruz Biotech (Santa Cruz, CA): anticaspase-3 (H-277), anti-DR4 (H130), anti-DR5 (N-19), anti-Mcl-1 (S-19), and anti-Bcl-2 (N-19). Annexin V-Cy5 was obtained from Biovision (Mountain View, CA). Anti-Myc (9B11), anti-caspase-8 (1C12), and anti-active caspase-3 (D175) were purchased from Cell Signaling (Beverly, MA). Anti-actin (clone $\mathrm{C} 4$ ) and anti- $\beta$-tubulin (clone AA2) were purchased from Millipore (Temecula, CA). Anti-human FLIP mAb (NF6) was purchased from AdipoGen (San Diego, CA). Horseradish peroxidaseconjugated secondary antibodies were purchased from Jackson ImmunoResearch Laboratories. WesternBright
ECL HRP substrate was obtained from Advansta Corporation (Menlo Park, CA). Dapi-Fluoromount-G ${ }^{\mathrm{TM}}$ was obtained from SouthernBiotech (Birmingham, AL). Protein G Mag Sepharose ${ }^{\mathrm{TM}}$ Xtra was obtained from GE Healthcare (Piscataway, NJ).

\section{Analysis of cancer gene microarray database}

The publicly-accessible Oncomine cancer microarray database (Compendia Biosciences; Ann Arbor, MI, USA; www.oncomine.com) was used to examine the expression of DTX1 in human gastric cancer tissue and cancer cell lines. D'Errico et al. (GEO accession GSE13911) ${ }^{43}$, Förster et al. (GEO accession GSE22377) ${ }^{44}$, and Ooi et al. (GEO accession GSE15459) ${ }^{45}$ datasets were used to compare 
DTX1, ITCH, and CBL expression levels between cancer and normal tissues. The Ooi et al. (GEO accession GSE15455) ${ }^{45}$ dataset was used to compare expression levels of $D T X 1, I T C H$, and $C B L$ among a panel of cell lines that represent adenocarcinoma, adenosquamous carcinoma, choriocarcinoma, and tubular adenocarcinoma of gastric cancer.

Hierarchical clustering analysis of gene expression data from 65 human primary tumor tissue samples and six gastrointestinal stromal tumor tissue samples was used to determine gene expression with gastric cancer outcomes $^{46}$. Primary microarray data (GEO accession GSE13891) were used to compare DTX1, ITCH, and CBL expression levels between $\mathrm{C} 1$ and $\mathrm{C} 2$ clusters.

\section{Cell culture and transfection}

AGS and SNU-16 cells were cultured in RPMI 1640 medium with $10 \%$ FCS (Life Technologies-Invitrogen), $10 \mathrm{mM}$ glutamine, $100 \mathrm{U} / \mathrm{ml}$ penicillin, $100 \mu \mathrm{g} / \mathrm{ml}$ streptomycin, and $20 \mathrm{mM}$ 2-ME. DMEM (Life Technologies) was used in the culture of 293T cells. Transfection of 293T cells was performed by using OmicsFect In Vitro Transfection Reagent (Omics Biotechnology, Taiwan).

\section{DTX1 overexpression and knockdown}

For overexpression of DTX1, mouse DTX1 cDNA (a gift from Dr. Hideyuki Okano, Keiko University, Tokyo, Japan) was tagged with FLAG and subcloned into pTRIPIRES-GFP to generate pTRIP-DTX1-IRES-GFP. 293T cells were transfected with pTRIP-IRES-GFP or pTRIP-c-FLIP-IRES-GFP, psPAX2, and pMD2G, and the lentivirus-containing culture supernatants were harvested $48 \mathrm{~h}$ after transfection. AGS and SNU-16 cells were infected with recombinant lentivirus, and GFP-expressing cells were isolated by sorting on a FACSAriaII SORP system (BD Biosciences). The c-FLIP p20 (198-376) fragment was isolated by PCR using forward primer $5^{\prime}$ ATG TCA AAT AAC TTC AGG CTC C and reverse primer 5 ' ATC CAC CTC CAA GAG GCT GC. A fulllength 2XHA-DTX1 (H453N and H456N, H2N2) mutant was generated ${ }^{40}$ and subcloned into pcDNA4 for expression in 293T cells.

For DTX1 knockdown, human DTX1-specific shRNA was subcloned into pLentiLox vector (pLL3.7; a gift from Dr. Luk Van Parijs, Massachusetts Institute of Technology, Cambridge, MA). The sequence of the human DTX1-specific shRNA was 5'-GAA GTT CAC CGC AAG AGG A-3'. Lentiviruses were harvested from culture supernatants of 293T cells transfected with pLL3.7 or pLL3.7-shDTX1, psPAX2, and pMD2.G. AGS and SNU-16 cells were infected with recombinant lentivirus, and GFP-expressing cells were sorted $48 \mathrm{~h}$ post-infection on a FACSAriaII SORP system (BD Biosciences, Mountain View, c-FLIP mouse [18] was a gift of Dr. You-Wen He (Duke University Medical Center, Durham $\mathrm{NC}$ ). Mice with $\mathrm{T}$ cell-specific knockout of c-FLIP were generated by crossing of c-FLIP mouse with Cd4-Cre mouse. Mouse experiments were conducted with approval from the Institutional Animal Care and Use Committee, Academia Sinica. CA).

\section{Apoptosis and cell viability assays}

Several different methods were used to measure apoptosis and viability. After TRAIL treatment for 5-6h, cells were stained with Annexin V-Cy5 and propidium iodide (PI), and annexin $\mathrm{V}^{+}$populations were quantified by flow cytometry. After TRAIL treatment for $24 \mathrm{~h}$, cells were stained with PI in hypotonic solution $(50 \mathrm{mg} / \mathrm{ml} \mathrm{PI}, 0.1 \%$ sodium citrate, $0.1 \%$ Triton $\mathrm{X}-100$ ) overnight at $4{ }^{\circ} \mathrm{C}$. Fractions of cells with sub-G1 DNA content were quantified using CellQUEST software on a FACSCalibur flow cytometer (BD Biosciences). For viability determination, AGS cells were plated in 96-well plates for $24 \mathrm{~h}$ before treatment. After TRAIL treatment, the cells were incubated with $0.5 \mathrm{mg} / \mathrm{ml} \mathrm{MTT} \mathrm{in} \mathrm{complete} \mathrm{medium} \mathrm{for} 2 \mathrm{~h}$. The surviving cells converted MTT to generate a purplecolored formazan product when dissolved in dimethyl sulfoxide (DMSO). The intensity of formazan product was measured by absorbance at $490 \mathrm{~nm}$ using SOFTmax PRO 4.3.1 LS software accompanying an Emax microtiter plate reader (Molecular Device, Sunnyvale, CA). Cell viability was calculated by dividing the absorbance of treated cells by that of the control. For measurement of caspase activation, control and DTX1-expressing cells were treated with $20 \mathrm{ng} / \mathrm{ml}$ TRAIL. The levels of procaspase-8, procaspase-3, processed caspase- $8(\mathrm{p} 43 / 41, \mathrm{p} 18)$ and processed caspase-3 (p19/17) were determined by immunoblots.

\section{Surface staining}

For staining of cell surface DR4 and DR5, AGS cells were incubated with anti-DR4 or anti-DR5 in PBS containing $2 \%$ FBS for $2 \mathrm{~h}$. After washing with PBS, cells were stained with allophycocyanin-conjugated anti-Rabbit IgG or anti-Goat IgG, and analyzed on a FACSCalibur flow cytometry system.

\section{Quantitative PCR}

Total RNA from AGS cells was isolated using TRIzol (Invitrogen). cDNAs were prepared and analyzed for the expression of DTX1, c-FLIP $\mathrm{L}_{\mathrm{L}}$ and c-FLIP $\mathrm{S}_{\mathrm{S}}$ on a LightCycler 480 Real-Time PCR System (Roche). The PCR protocol was $95^{\circ} \mathrm{C}$ for $10 \mathrm{~min}$, followed by 45 cycles of $95^{\circ} \mathrm{C}$ for $10 \mathrm{~s}$, $60^{\circ} \mathrm{C}$ annealing for $10 \mathrm{~s}$, and $72^{\circ} \mathrm{C}$ extension for $8 \mathrm{~s}$. The PCR primers were: human DTX1, forward, $5^{\prime}$ CAG CCG 
CCT GGG AAG ATG GAG TT-3' and reverse, 5' ATG CCT GTG GGG ATG TCA TAG AC-3'; human cFLIP $_{\mathrm{L}}$, forward, 5'-CCT AGG AAT CTG CCT GAT AAT CGA-3' and reverse, $5^{\prime}$-TGG GAT ATA CCA TGC ATA CTG AGA TG-3'; human c-FLIP , forward, 5'-GCA GCA ATC CAA AAG AGT CTC A $-3^{\prime}$ and reverse, 5'-ATT TCC AAG AAT TTT CAG ATC AGG A-3'.

\section{Immunoprecipitation}

Cells were washed and lysed in whole cell extract (WCE) buffer (25 mM HEPES $\mathrm{pH} 7.4,300 \mathrm{mM} \mathrm{NaCl}$, $1.5 \mathrm{mM} \mathrm{MgCl}_{2}, 0.2 \mathrm{mM}$ EDTA pH 8.0, 0.1\% Triton X-100, $0.5 \mathrm{mM}$ DTT, $1 \times$ Complete protease inhibitor (EDTAfree, Roche)). Two hundred and fifty microgram whole cell lysate was incubated with antibodies, as indicated in each figure, on a rotating shaker overnight at $4{ }^{\circ} \mathrm{C}$. Five microliter of Protein G Mag Sepharose was added and the samples were incubated at $4{ }^{\circ} \mathrm{C}$ for $1 \mathrm{~h}$. The Protein G Mag Sepharose was washed repeatedly with $1 \mathrm{ml}$ of WCE buffer. The beads were mixed with $1 \times$ SDS-PAGE sample buffer and boiled at $100^{\circ} \mathrm{C}$ for $5 \mathrm{~min}$. Pull-down complex and total lysates were analyzed in 10\% SDS-PAGE and transferred to polyvinylidene difluoride (PVDF) membranes (Millipore). The membranes were blocked with 5\% skimmed milk for $1 \mathrm{~h}$ and probed with an appropriate antibody, followed by incubation with a HRP-conjugated secondary antibody. The protein bands were visualized by the ECL detection system.

\section{Protein stability determination}

c-FLIP $\mathrm{L}_{\mathrm{L}}$-Myc was transfected with or without DTX1Flag in 293T cells. Cells were treated with cycloheximide (CHX, $50 \mathrm{ng} / \mathrm{ml}$ ) for the indicated timeframes. Cell lysates were prepared and contents of c-FLIP $\mathrm{L}_{\mathrm{L}}-\mathrm{Myc}$ and DTX1Flag were quantitated. The direct effect of DTX1 on cFLIP $_{\mathrm{L}}$ protein levels was examined by transfecting 293T cells with c-FLIP or $_{\text {c-FLIP }}$ in the presence of increasing amounts of DTX1. The levels of DTX1, cFLIP $_{\mathrm{L}}$ and c-FLIP $\mathrm{S}$ were determined $24 \mathrm{~h}$ after transfection. To identify the inhibitor of c-FLIP degradation, DTX1-Myc and/or c-FLIP ${ }_{\mathrm{L}}$-Flag were transfected into 293T cells, and cells were treated with or without MG132 $(2.5 \mu \mathrm{M}), \mathrm{NH}_{4} \mathrm{Cl}(25 \mathrm{mM})$, or leupeptin $(100 \mu \mathrm{g} / \mathrm{ml})$. The levels of c-FLIP ${ }_{\mathrm{L}}$-Flag and DTX1-Myc were determined $8 \mathrm{~h}$ later.

\section{Fluorescence protein constructs and confocal imaging}

pmRFP-Rab5 was obtained from Addgene (Addgene plasmid 14437). pcDNA4-EGFP-DTX1 and pcDNA4LAMP1-mCherry were generated as described previously ${ }^{9}$. To generate pcDNA4-cerulean-c-FLIP ${ }_{\mathrm{L}}$, cerulean fragments were amplified by PCR from Cerulean-GalT (Addgene plasmid 11930) with HindIII/
EcoRI and cloned into pcDNA4-c-FLIP ${ }_{\mathrm{L}}$-Myc with HindIII/EcoRI to create a translational fusion construct.

293T cells $\left(1 \times 10^{6}\right)$ transduced with mRFP-Rab5 or LAMP1-mCherry were transfected with cerulean-c-FLIP ${ }_{\mathrm{L}}$ and EGFP-DTX1. $24 \mathrm{~h}$ after transfection, cells were reseeded on a $22 \times 22 \mathrm{~mm}$ glass coverslip and allowed to attach for another $18 \mathrm{~h}$. Cells were fixed in $2 \%$ paraformaldehyde in PBS for $15 \mathrm{~min}$ at $37^{\circ} \mathrm{C}$ and permeabilized with $0.3 \%$ Triton $\mathrm{X}-100$ for $10 \mathrm{~min}$ at room temperature. Cells were mounted in Dapi-Fluoromount$\mathrm{G}^{\mathrm{TM}}$ (SouthernBiotech, Birmingham, AL) and observed under a Zeiss LSM 780 confocal microscope.

\section{Image acquisition}

Images were observed under a Zeiss LSM 780 confocal microscope with a plan-Apochromat $63 \times / 1.4$ Oil DIC objective lens at room temperature. Samples were mounted in Dapi-Fluoromount-G ${ }^{\mathrm{TM}}$. EGFP and Cerulean fluorescence proteins were excited by an argon laser (488 $\mathrm{nm}$ ). EGFP fluorescence was collected in the range of 490 to $550 \mathrm{~nm}$. Cerulean fluorescence was collected in the range of 445 to $489 \mathrm{~nm}$. mRFP and mCherry fluorescence proteins were excited by a $\mathrm{HeNe}$ laser $(594 \mathrm{~nm})$ and emissions were collected with a $545 \mathrm{~nm}$ long-pass filter. DAPI-bound DNA was excited by a diode laser $(405 \mathrm{~nm})$ and fluorescence was collected in the range of 405 to 470 $\mathrm{nm}$. The pinholes were as follows: Ch1-55 $\mu \mathrm{m}$ (blue), Chs1-54 $\mu \mathrm{m}$ (orange), Ch2-55 $\mu \mathrm{m}$ (red), and Chs1-55 $\mu \mathrm{m}$ (green). Images were acquired by a digital AxioCam (Zeiss) microscope camera using Carl Zeiss software Zen 2.1 (black).

\section{Acknowledgements}

We thank Ms. Yamin Lin of the FACS Core and Shu-Mei Huang of the Confocal Core of the Institute of Molecular Biology, Academia Sinica for cell sorting and confocal microscopy, and Dr. John O'Brien for editing the manuscript. This work was supported by grant MOST 105-2321-B-001-065 from the Ministry of Science and Technology and an Academia Sinica Investigator Award from Academia Sinica, Taiwan, R.O.C.

\section{Author contributions}

T.S.H.: acquisition, analysis and interpretation of data, statistical analyses; S.T.M.: data acquisition; P.N.H.: material support and study concept; M.Z.L.: study concept and design, supervision of the study, drafting of the manuscript.

\section{Conflict of interest}

The authors declare that they have no conflict of interest.

\section{Publisher's note}

Springer Nature remains neutral with regard to jurisdictional claims in published maps and institutional affiliations.

\section{Supplementary information}

The online version of this article (https://doi.org/10.1038/s41419-017-0165-6) contains supplementary material.

Received: 2 August 2017 Revised: 17 November 2017 Accepted: 17 November 2017

Published online: 26 January 2018 


\section{References}

1. Ferlay, J. et al. Cancer incidence and mortality worldwide: sources, methods and major patterns in GLOBOCAN 2012. Int. J. Cancer 136, E359-E386 (2015).

2. Corso, S. \& Giordano, S. How can gastric cancer molecular profiling guide future therapies? Trends Mol. Med. 22, 534-544 (2016).

3. Van Cutsem, E., Sagaert, X., Topal, B., Haustermans, K. \& Prenen, H. Gastric cancer. Lancet 388, 2654-2664 (2016)

4. Ashkenazi, A. Targeting the extrinsic apoptotic pathway in cancer: lessons learned and future directions. J. Clin. Invest. 125, 487-489 (2015).

5. Lim, B. et al. Targeting TRAIL in the treatment of cancer: new developments. Expert Opin. Ther. Targets 19, 1171-1185 (2015).

6. von Karstedt, S., Montinaro, A. \& Walczak, H. Exploring the TRAlls less travelled: TRAIL in cancer biology and therapy. Nat. Rev. Cancer 17, 352-366 (2017).

7. Qiao, L. \& Wong, B. C. Targeting apoptosis as an approach for gastrointestinal cancer therapy. Drug Resist. Updat. 12, 55-64 (2009).

8. Huang, Y. et al. sTRAlL-iRGD is a promising therapeutic agent for gastric cancer treatment. Sci. Rep. 7, 579 (2017).

9. Strasser, A., Jost, P. J. \& Nagata, S. The many roles of FAS receptor signaling in the immune system. Immunity 30, 180-192 (2009).

10. Wilson, N. S., Dixit, V. \& Ashkenazi, A. Death receptor signal transducers: nodes of coordination in immune signaling networks. Nat. Immunol. 10, 348-355 (2009).

11. Lavrik, I. N. \& Krammer, P. H. Regulation of CD95/Fas signaling at the DISC. Cell Death Differ. 19, 36-41 (2012).

12. Green, D. R. \& Llambi, F. Dell death signaling. Cold Spring Harb. Perspect. Biol. 7 a006080 (2015)

13. Budd, R. C., Yeh, W. C. \& Tschopp, J. cFLIP regulation of lymphocyte activation and development. Nat. Rev. Immunol. 6, 196-204 (2006).

14. Hughes, M. A. et al. Co-operative and hierarchical binding of C-FLIP and Caspase-8: a unified model defines how c-FLIP isoforms differentially control cell fate. Mol. Cell. 61, 834-849 (2016)

15. Schleich, K. et al. Molecular architecture of the DED chains at the DISC: regulation of procaspase-8 activation by short DED proteins C-FLIP and procaspase-8 prodomain. Cell Death Differ. 23, 681-694 (2016).

16. Feoktistova, M. et al. CIAPs block Ripoptosome formation, a RIP1/caspase-8 containing intracellular cell death complex differentially regulated by cFLIP isoforms. Mol. Cell. 43, 449-463 (2011).

17. Oberst, A. et al. Catalytic activity of the caspase-8-FLIP complex inhibits RIPK3dependent necrosis. Nature 471, 363-367 (2011).

18. He, M. X. \& He, Y. W. A role for C-FLIP in the regulation of apoptosis, autophagy, and necroptosis in T lymphocytes. Cell Death Differ. 20, 188-197 (2013).

19. Fulda, S. Targeting extrinsic apoptosis in cancer: Challenges and opportunities. Semin. Cell. Dev. Biol. 39, 20-25 (2015).

20. Safa, A. R. \& Pollok, K. E. Targeting the anti-apoptotic protein c-FLIP for cancer therapy. Cancers 3, 1639-1671 (2011).

21. Shirley, S. \& Micheau, O. Targeting C-FLIP in cancer. Cancer Lett. 332, 141-150 (2013).

22. Yeh, J. H., Hsu, S. C., Han, S. H. \& Lai, M. Z. Mitogen activated protein kinase kinase antagonized FADD-mediated apoptosis by induced FLIP expression. J. Exp. Med. 188, 1795-1802 (1998).

23. Micheau, O., Lens, S., Gaide, O., Alevizopoulos, K. \& Tschopp, J. NF-kB signals induce the expression of c-FLIP. Mol. Cell. Biol. 21, 5299-5305 (2001)

24. Kreuz, S., Siegmund, D., Scheurich, P. \& Wajant, H. NF-KB inducers upregulate CFLIP, a cycloheximide-sensitive inhibitor of death receptor signalling. Mol. Cell. Biol. 21, 3964-3973 (2001)

25. Chang, L. et al. The E3 ubiquitin ligase itch couples JNK activation to TNFalphainduced cell death by inducing c-FLIP(L) turnover. Cell 124, 601-613 (2006).

26. Zhao, L., Yue, P., Khuri, F. R. \& Sun, S. Y. mTOR complex 2 is involved in regulation of Cbl-dependent c-FLIP degradation and sensitivity of TRAILinduced apoptosis. Cancer Res. 73, 1946-1957 (2013)

27. Koyama, S., Koike, N. \& Adachi, S. Expression of TNF-related apoptosis-inducing ligand (TRAIL) and its receptors in gastric carcinoma and tumor-infiltrating lymphocytes: a possible mechanism of immune evasion of the tumor. $J$. Cancer Res. Clin. Oncol. 128, 73-79 (2002).

28. Kanehara, I., Nakata, B. \& Hirakawa, K. Caspase-8 is scarcely silenced and its activity is well correlated with the anticancer effect of tumor necrosis factorrelated apoptosis-inducing ligand in gastric cancer cells. Oncol. Rep. 14 1249-1253 (2005)
29. Lee, S. H. et al. Increased expression of FLIP, an inhibitor of Fas-mediated apoptosis, in stomach cancer. APMIS 111, 309-314 (2003).

30. Zhou, X. D. et al. Overexpression of cellular FLICE-inhibitory protein (FLIP) in gastric adenocarcinoma. Clin. Sci. 106, 397-405 (2004).

31. Nam, S. Y. et al. Upregulation of FLIP(S) by Akt, a possible inhibition mechanism of TRAlL-induced apoptosis in human gastric cancers. Cancer Sci. 94, 1066-1073.

32. Wang, W. et al. Programmed cell death 4 (PDCD4) mediates the sensitivity of gastric cancer cells to TRAlL-induced apoptosis by down-regulation of FLIP expression. Exp. Cell. Res. 316, 2456-2464 (2010).

33. Belkhiri, A., Zhu, S., Chen, Z, Soutto, M. \& El-Rifai, W. Resistance to TRAlL is mediated by DARPP-32 in gastric cancer. Clin. Cancer Res. 18, 3889-3900 (2012).

34. Lin, W. C. et al. Helicobacter pylori sensitizes TNF-related apoptosis-inducing ligand (TRAIL)-mediated apoptosis in human gastric epithelial cells through regulation of FLIP. Cell Death Dis. 5, e1109 (2014).

35. Matsuno, K., Diederich, R. J., Go, M. J., Blaumueller, C. M. \& Artavanis-Tsakonas, S. DTX acts as a positive regulator of Notch signaling through interactions with the Notch ankyrin repeats. Development 121, 2633-2644 (1995).

36. Kishi, N. et al. Murine homologs of DTX define a novel gene family involved in vertebrate Notch signaling and neurogenesis. Int. J. Dev. Neurosci. 19, 21-35 (2001).

37. Hori, K. et al. Drosophila Deltex mediates Suppressor of Hairless-independent and late-endosomal activation of Notch signaling. Development 131, 5527-5537 (2004)

38. Hori, K., Sen, A., Kirchhausen, T. \& Artavanis-Tsakonas, S. Synergy between the ESCRT-III complex and Deltex defines a ligand-independent Notch signal. J. Cell. Biol. 195, 1005-1015 (2011)

39. Heissmeyer, $V$. et al. Calcineurin imposes $T$ cell unresponsiveness through targeted proteolysis of signaling proteins. Nat. Immunol. 5, 255-265 (2004).

40. Hsiao, H. W. et al. Deltex1 is a target of the transcription factor NFAT that promotes T cell anergy. Immunity 31, 72-83 (2009).

41. Hsiao, H. W. et al. Deltex 1 antagonizes HIF-1a and sustains the stability of regulatory T cells in vivo. Nat. Commun. 6, 6353 (2015)

42. Hsu, T. S., Hsiao, H. W., Wu, P. J., Liu, W. H. \& Lai, M. Z. Deltex1 promotes protein kinase $C \theta$ degradation and sustains Casitas B-lineage lymphoma expression. J. Immunol. 193, 1672-1680 (2014)

43. D'Errico, M. et al. Genome-wide expression profile of sporadic gastric cancers with microsatellite instability. Eur. J. Cancer 45, 461-469 (2009).

44. Förster, S., Gretschel, S., Jöns, T., Yashiro, M. \& Kemmner, W. THBS4, a novel stromal molecule of diffuse-type gastric adenocarcinomas, identified by transcriptome-wide expression profiling. Mod. Pathol. 24, 1390-1403 (2011).

45. Ooi, C. H. et al. Oncogenic pathway combinations predict clinical prognosis in gastric cancer. PLoS Genet. 5, e1000676 (2009).

46. Cho, J. Y. et al. Gene expression signature-based prognostic risk score in gastric cancer. Clin. Cancer Res. 17, 1850-1857 (2011)

47. Wang, Q. et al. Down-regulation of cellular FLICE-inhibitory protein (Long Form) contributes to apoptosis induced by Hsp90 inhibition in human lung cancer cells. Cancer Cell. Int. 12, 54 (2012).

48. Panner, A., Murray, J. C., Berger, M. S. \& Pieper, R. O. Heat shock protein 90alpha recruits FLIPS to the death-inducing signaling complex and contributes to TRAlL resistance in human glioma. Cancer Res. 67, 9482-9489 (2007).

49. Whitesell, L. \& Lin, N. U. Hsp90 as a platform for the assembly of more effective cancer chemotherapy. Biochim. Biophys. Acta 1823, 756-766 (2012).

50. Vasilevskaya, I. A. \& O'Dwyer, P. J. 17-Allylamino-17-demethoxygeldanamycin overcomes TRAlL resistance in colon cancer cell lines. Biochem. Pharmacol. 70 580-589 (2005)

51. Wang, $X$ et al. 17-allylamino-17-demethoxygeldanamycin synergistically potentiates tumor necrosis factor-induced lung cancer cell death by blocking the nuclear factor-kappaB pathway. Cancer Res. 66, 1089-1095 (2006).

52. Henrich, C. J. et al. Withanolide E sensitizes renal carcinoma cells to TRAlLinduced apoptosis by increasing cFLIP degradation. Cell Death Dis. 6, e1666 (2015).

53. Chen, H., Li, L. Q. \& Pan, D. Geldanamycin induces apoptosis in human gastric carcinomas by affecting multiple oncogenic kinases that have synergic effects with TNF-related apoptosis-inducing ligand. Oncol. Lett. 10, 3732-3736 (2015). 